\title{
Diseño e implementación de una aplicación móvil de realidad aumentada, para la enseñanza de química general en ingeniería
}

\section{Design and implementation of an augmented reality mobile application, for teaching general chemistry in engineering}

DOI: $10.46932 / \mathrm{sfjdv2n4-096}$

Received in: March 1st, 2021

Accepted in: May 30th, 2021

Andrea de las Mercedes Arias Padilla

Ingeniero en Alimentos de la Universidad de La Frontera-Chile, Magíster en Didáctica de las Ciencias Experimentales de la Universidad Católica de Valparaíso-Chile Institución: la Universidad de La Frontera-Chile

Dirección: Av. Francisco Salazar 01145, Temuco-Chile

E-mail: andrea.arias@ufrontera.cl

\section{Tania Gloria Tapia Opazo}

Químico de la Pontificia Universidad Católica de Chile, Magister en Ciencias de Recursos Naturales de

la Universidad de La Frontera-Chile

Institución: Universidad de La Frontera-Chile

Dirección: Av. Francisco Salazar 01145, Temuco-Chile

E-mail: tania.tapia@ufrontera.cl

Natacha Alejandra Pino Acuña

Ingeniera Civil Industrial de la Universidad de La Frontera-Chile, Magister en Ingeniería de la

Universidad de Valencia-España

Institución: Universidad de Aysén-Chile

Dirección: Obispo Vielmo 62, Coyhaique-Chile

E-mail: natacha.pino@uaysen.cl

\section{Juan José Gutiérrez Álvarez}

Psicólogo y Magister en Ciencias Sociales Aplicadas de la Universidad de La Frontera. Institución: Centro de Innovación y Emprendimiento Kowork Chile SpA.

Dirección: Av. Canadá 1553, Ciudad Primavera, Vilcún-Chile

E-mail: juanjo.gutierrez.a@gmail.com

\section{RESUMEN}

La química es una ciencia básica que incluye conceptos abstractos, fenómenos y objetos que no pueden verse a simple vista, lo que dificulta un aprendizaje significativo y homogéneo en los estudiantes, además de obstaculizar la motivación en el proceso de aprendizaje de esta ciencia. Por lo anterior, este trabajo propone como objetivo general el diseño e implementación de una aplicación móvil con tecnología de Realidad Aumentada (RA) para utilizar como apoyo en el proceso de enseñanza-aprendizaje de la Química General en carreras de ingeniería de la Universidad de La Frontera, y que nos permita determinar el grado de motivación que genera en los estudiantes.

La propuesta, tiene como primera etapa el diseño de la aplicación móvil con uso de RA en la temática de Equilibrio ácido-base, utilizando el motor de videojuegos multiplataforma Unity y el SDK de Vuforia, y una segunda etapa de prueba de la implementación a estudiantes de primer año de Ingeniería, donde además se aplicó una encuesta de percepción. En esta última, los estudiantes manifestaron una gran 
aceptación de la aplicación, además de señalar que la incorporación de nuevas tecnologías en la enseñanza de la química permite una mayor motivación e interés en la clase.

Palabras clave: Realidad aumentada, Enseñanza de la química, Motivación

\begin{abstract}
Chemistry is a basic science that includes abstract concepts, phenomena and objects that can not be seen with the naked eye. That makes more difficult for the students to obtain a meaningful and homogeneous learning, and makes more difficult to get motivation in the Chemistry learning process. That is why the general objective of this work is the design and implementation of an application using augmented reality in order to use the application as a complementary material in the Chemistry teaching-learning process, in the subject General Chemistry for engineering careers in Universidad de la Frontera, and to determinate how motivated the students can be after using this application.

The first phase in this process is the designing of the application using augmented reality with the subject acid-base balance, using the videogames multiplatform motor Unity and the Vuforia's SDK. The second phase was testing the application on first year engineer students in Universidad de la Frontera, it was also added a poll to have an opinion of them. They were satisfied with the application and declared that the use of new technologies increases the motivation for the subject.
\end{abstract}

Keywords: augmented reality, chemistry teaching, motivation

\title{
1 JUSTIFICACIÓN
}

Actualmente las ciencias básicas son fundamentales en la formación integral de un ingeniero, por lo que la didáctica de las ciencias tiene la responsabilidad de provocar profundos cambios en los diferentes elementos del currículum y la metodología de la enseñanza, con el fin de lograr que los cursos se desarrollen vinculados con la realidad y que los estudiantes aprendan lo indicado (Torres, 2010).

La química, es una ciencia con características propias, con métodos basados en el análisis y la síntesis, que debe ser razonada y no aprendida de memoria; una sólida base teórica y la resolución de problemas resulta imprescindible. El docente entonces debe orientarse a desarrollar las habilidades y destrezas en el aprendizaje de los estudiantes para acrecentar el interés y la motivación (Antezana, 2009).

Es en este contexto, que la actividad docente debe priorizar la incorporación de nuevas metodologías en el proceso de enseñanza-aprendizaje y así estimular la participación activa de los estudiantes y mejorar sus rendimientos. Para tener éxito en este proceso, no sólo basta con mostrar un contenido desde otro ángulo, sino que, además es necesario considerar los distintos tipos de aprendizaje que posee cada estudiante (Acevedo et al., 2015).

El informe Horizon (2010), describe las tecnologías emergentes que tendrán un fuerte impacto en la docencia, el aprendizaje o la investigación creativa en facultades y campus universitarios en los próximos años, se incluye la Realidad Aumentada (RA) prediciendo un horizonte de implantación de pocos años y considera que tiene un gran potencial para proporcionar tanto experiencias de aprendizaje 
contextual e in situ valiosas, como de exploración y descubrimiento fortuito de la información conectada en el mundo real (Martínez-Hung et al., 2017).

La Realidad Aumentada (RA) es la combinación de ambientes reales, a los cuales se incorpora información en formato digital con el fin de ampliar lo que nuestros sentidos captan sobre situaciones de la realidad. Esto se puede visualizar en una pantalla donde se mezclan la realidad captada por una cámara (en tiempo real) y la información virtual creada previamente y sincronizada a través de marcas (p. ej., tarjetas con dibujos o diagramas en blanco y negro) o por posicionamiento geográfico (vinculado al uso de internet) (Merino et al., 2015).

Entre las ventajas que Merino et al. (2015) han identificado con el uso de la RA, se puede mencionar:

A. Facilita la enseñanza de conceptos abstractos, fenómenos y objetos que no pueden verse a simple vista.

B. Permite establecer un puente entre los conceptos teóricos y la realización física de los experimentos con los dispositivos reales.

C. Los estudiantes, al interactuar con RA, movilizan los mismos recursos cognitivos que usarían para el tratamiento con objetos de la vida real, centrando su atención en el objeto de aprendizaje y no el objeto mediador.

\section{OBJETIVO DEL PROYECTO DE INNOVACIÓN}

Diseñar e implementar una aplicación móvil con tecnología de Realidad Aumentada (RA) para utilizar como apoyo en el proceso de enseñanza-aprendizaje de la Química General en carreras de ingeniería de la Universidad de La Frontera, y que nos permita determinar el grado de motivación que genera en los estudiantes.

\section{METODOLOGÍA O PROPUESTA DE ACTUACIÓN}

La metodología de trabajo contempla 2 fases: Diseño de una aplicación móvil de RA y su implementación a estudiantes de primer año de Ingeniería de la Universidad de La Frontera.

\section{Fase 1: Diseño de la aplicación}

\section{Elección del contenido}

Para determinar en qué contenidos enfocar la aplicación, se consideraron los resultados de los diagnósticos de ciencias básicas que los estudiantes de primer año de la Facultad de Ingeniería y Ciencias deben rendir al ingresar a la universidad. 
El diagnóstico de química fue respondido por 570 estudiantes de ingreso 2018. Según el análisis de los resultados, en promedio los estudiantes presentaron un 50,96\% de aciertos en sus respuestas. En la Tabla $\mathrm{N}^{\mathrm{o}}$ 1, se presentan los rendimientos según dominios conceptuales (taxonomías) donde se observa que los contenidos más débiles se asocian a las temáticas de Equilibrio Ácido-base, presentando un $48,07 \%$ de aciertos.

Tabla N ${ }^{\circ} 1$ Rendimiento diagnóstico de química ingreso 2018 (Fuente: elaboración propia).

\section{TAXONOMÍA}

1. Teoría atómica y estructura electrónica

2. Tabla periódica y sus propiedades

3. Reacciones químicas y estequiometría

4. Propiedades de soluciones

5. Equilibrio ácido-base
ACIERTOS

$53,24 \%$

$\mathbf{5 0 , 0 2 \%}$

$51,35 \%$

$52,12 \%$

$48,07 \%$

De acuerdo a estos resultados, se determinó aplicar el prototipo de RA en torno a la unidad temática de Equilibrio Ácido-base, particularmente en el contenido de Indicadores de $\mathrm{pH}$.

\section{Diseño del prototipo de la aplicación de RA}

- Generación de modelos tridimensionales que representen los contenidos requeridos en soluciones indicadoras utilizando el Software BLENDER.

- Elaboración de marcadores de reconocimiento de patrones para los modelos tridimensionales.

- Programación de las interacciones entre los modelos tridimensionales a través del Motor Gráfico UNITY y el SDK de VUFORIA.

La siguiente etapa fue la incorporación al prototipo de las soluciones indicadoras de $\mathrm{pH}$. Para ello se seleccionaron 3 indicadores: fenolftaleína, naranja de metilo y azul de timol (Tabla $\mathrm{N}^{\mathrm{o}} 2$ ). Lo anterior, se realizó considerando soluciones indicadoras con virajes de color en medio ácido y básico claramente observables.

Tabla $N^{\circ} 2$ Intervalo de viraje de color de indicadores (Fuente: elaboración propia)

\begin{tabular}{|c|c|c|c|c|}
\hline Solución & $\mathbf{p H}$ & $\begin{array}{l}\text { Fenolftaleína } \\
(\text { pH 8,2-10,0) }\end{array}$ & $\begin{array}{l}\text { Azul de timol } \\
(\mathrm{pH} 8,0-9.6)\end{array}$ & $\begin{array}{l}\text { Naranja de metilo } \\
\text { (pH 3,1-4,4) }\end{array}$ \\
\hline Ácido clorhídrico $(\mathrm{HCl})$ & 1,1 & Incoloro & Rojo & Rojo \\
\hline $\begin{array}{l}\text { Ácido acético } \\
\left(\mathrm{CH}_{3} \mathrm{COOH}\right)\end{array}$ & 2,9 & Incoloro & Rojo & Rojo \\
\hline $\begin{array}{l}\text { Bicarbonato de sodio } \\
(\mathrm{NaHCO})\end{array}$ & 8,0 & Rosado & Azul & Amarillo \\
\hline Hidróxido de sodio & 12,9 & Rosado & Azul & Amarillo \\
\hline
\end{tabular}


$(\mathrm{NaOH})$

El indicador se agregó al prototipo a través de la activación de una animación por medio de botones ubicados en un menú lateral derecho, tal como se muestra en la Figura $\mathrm{N}^{\circ} 1$.

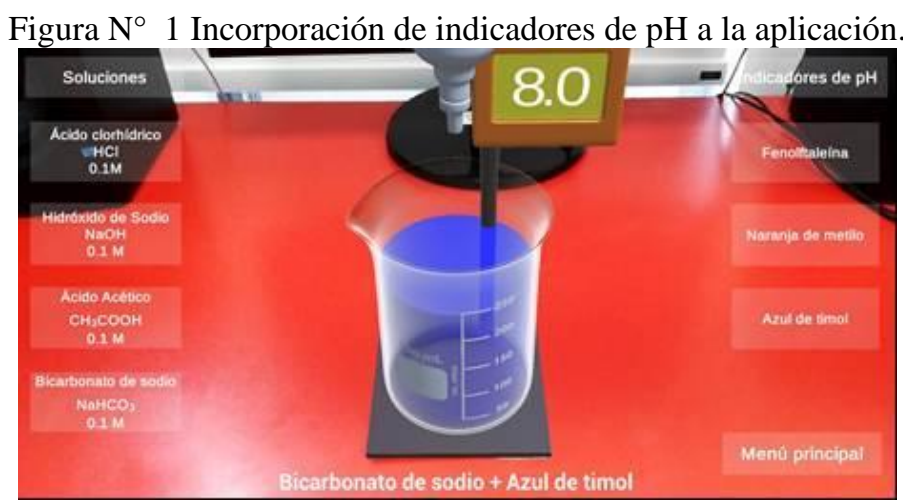

Fuente: propia

\section{Pilotaje}

Se realizó una prueba piloto del prototipo para poder incorporar los ajustes necesarios al diseño de la aplicación. En esta prueba piloto participaron de forma voluntaria 20 estudiantes de la Facultad de Ingeniería y Ciencias de la Universidad de La Frontera, los cuales debían utilizar dispositivos móviles (smartphones) con sistema operativo Android, para visualizar la actividad práctica virtual.

\section{Fase 2: Implementación de la aplicación}

\section{Aplicación de la innovación en aula}

La innovación se aplicó a 78 estudiantes de primer año de las carreras de Ingeniería Civil e Ingeniería en Construcción. En primera instancia, se aplicó a los estudiantes un diagnóstico asociado al uso de TIC, con una encuesta de 6 preguntas cerradas, de las cuales cuatro de ellas dependiendo de la respuesta se le pedía explicar el porqué de esa respuesta, con estas preguntas se buscaba conocer alguna otra información respecto del uso de tecnología y en especial RA. En una segunda etapa, los estudiantes bajaron la aplicación en sus dispositivos móviles y luego con el uso de un marcador pudieron activar los elementos virtuales del diseño. Durante la actividad, los estudiantes debieron completar una guía de trabajo asociada al contenido. 


\section{RESULTADOS}

\section{Fase 1. Diseño de la aplicación}

\section{Diseño del prototipo de la aplicación de RA}

- Desarrollo de la aplicación: Se utilizó el motor de videojuegos multiplataforma Unity y el SDK de Vuforia, el kit de desarrollo de software de RA. En Unity se diseñó la mecánica de funcionamiento de la aplicación. Por su parte, Vuforia se utilizó para calcular la posición de un marcador a través de la cámara del smartphone e integrar los objetos tridimensionales en la escena. Cuando el marcador es detectado por la cámara, la fusión del mundo real y los objetos virtuales se muestra en la pantalla del smartphone.

- Marcadores: Para la activación de los elementos virtuales se utilizó un marcador NFT (Natural Feature Tracking o Seguimiento de Características Naturales, que en nuestro caso, luego de diferentes pruebas de estabilidad, velocidad de reconocimiento y oclusión, se seleccionó la fotografía de un liquen rojo (Figura $\mathrm{N}^{\mathrm{o}}$ 2) (organismo que surge de la simbiosis entre un hongo y un alga), esto dado las características de la imagen, debido a que no corresponde a un patrón repetitivo y sus componentes son perfectamente distinguibles unos de otros. Por otra parte, el marcador fue impreso en un material opaco, para que no interfiriera el reflejo de la iluminación. Además, se optó por proporcionar este marcador en un tamaño menor, 6 × $6 \mathrm{~cm}$, para que se adecuara al campo de visión de la cámara del smartphone y fuera fácilmente manipulable para el usuario.

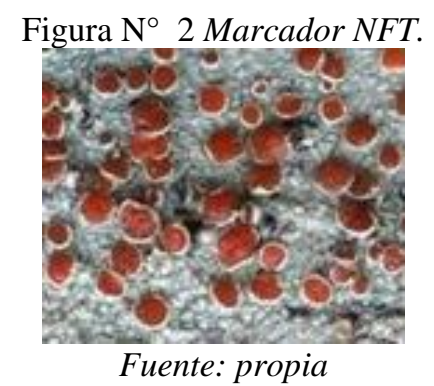

- Funcionalidad de la aplicación: Para controlar las secuencias de animación y el acceso a menús, se programaron scripts en el lenguaje de programación orientado a objetos C\#. Se compiló la aplicación en formato apk, para el sistema operativo Android desde la versión 4.1 “Jelly Bean”. La Figura $N^{\circ} 3$ muestra el flujo de trabajo del diseño de la aplicación. 
Figura $\mathrm{N}^{\circ} 3$ Flujo de trabajo del diseño.

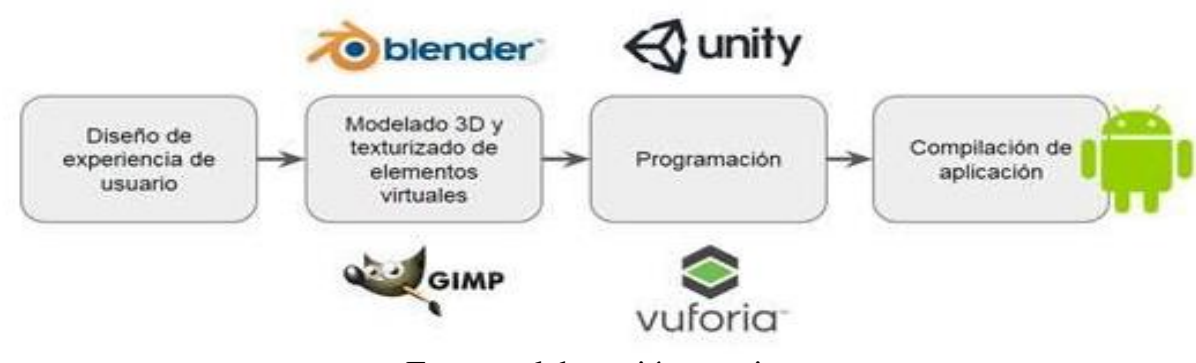

Fuente: elaboración propia

\section{Pilotaje}

Una vez aplicado el diagnóstico, la encuesta mostró que el 83,33\% de los estudiantes conoce las TICs y que las más utilizadas por ellos son: Word, Powerpoint, Excel, Google drive, Dispositivos móviles y Notebook. Sin embargo, solo el 33,33\% señala conocer la tecnología de RA y en general la relacionan sólo a actividades de entretención o recreativas, no a contextos educativos o de aprendizaje.

Finalmente, los estudiantes al terminar el pilotaje realizaron comentarios respecto a la actividad (pregunta abierta al final de la guía de trabajo). Al analizar las respuestas, se observó que todos los estudiantes opinaron que la actividad fue interesante y que generó una dinámica más activa con sus compañeros: (1) "La actividad es novedosa, genera interés y motivación por realizarla", (2) "Es maravillosa la aplicación” у (3) “Me pareció interesante, bonita y llamativa. El que sea del tipo realidad aumentada la hace mucho más novedosa e interactiva".

Además, los estudiantes señalan que la aplicación con uso de tecnología de RA es adecuada como herramienta de aprendizaje en contenidos de química, reflejado de manera descriptiva en sus comentarios: (1) "La actividad me parece muy entretenida y un buen acercamiento para los estudiantes que no tengan clases en laboratorio”, (2) “La encuentro muy útil, ya que es fácil de entender cómo se produce el cambio de color en los indicadores dependiendo del pH" у (3) "Es una actividad muy entretenida, permite observar claramente los cambios de color cuando se mezcla un indicador con una solución determinada". Esto concuerda con estudios en asignaturas de química a nivel universitario donde se aplicó tecnología de RA y la valoración de los estudiantes fue positiva, ya que la misma contribuyó a incrementar el interés y comprensión de los contenidos fundamentalmente en la representación de estructuras moleculares, orbitales y elementos de simetría (Martínez y col., 2017).

Todo lo anterior, coincide con lo señalado por Saidin et al. (2015), referente a que la tecnología RA tiene un potencial positivo y ventajas que se pueden adaptar en la educación para hacer que el proceso de aprendizaje sea más activo, efectivo y significativo. Esto debido a que genera una mayor disposición de los estudiantes a participar activamente en sus estudios, involucra a los estudiantes en los procesos de aprendizaje y ayuda a mejorar sus habilidades de visualización. Sin embargo, existen limitaciones en el 
uso de esta tecnología y se relacionan principalmente con problemas técnicos y con la necesidad de actualización permanente de los docentes.

\section{Fase 2. implementación de la aplicación}

\section{Aplicación de la innovación en aula}

El diagnóstico inicial aplicado señala que 49 de los 78 estudiantes (63\%) conoce las tecnologías de información y comunicación. Sin embargo, al momento de preguntar si la utilizan, solamente $27 \%$ reconoce utilizar a menudo alguna herramienta TIC. Al asociar el uso de tecnología como apoyo didáctico en los procesos de enseñanza aprendizaje, prácticamente el 100\% de los estudiantes consideran que es un recurso útil.

Figura $\mathrm{N}^{\circ} 4$ Gráfico respecto al conocimiento de la tecnología.

\section{¿Conoce las Tecnologías de Información y}

Comunicación (TIC)?.

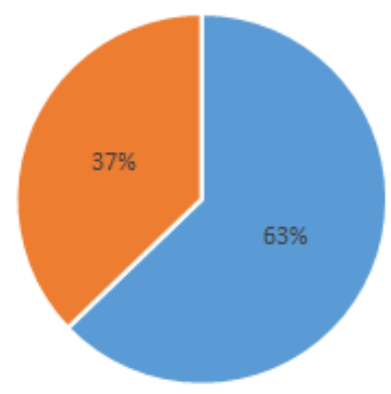

ni $=$ No

Fuente: propia

Figura $\mathrm{N}^{\circ} 5$ Gráfico respecto a la utilización de tecnologia.

¿Ha utilizado alguna TIC?.

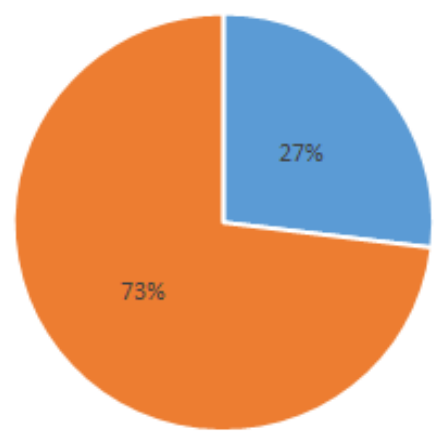

- Si $=$ No

Fuente: propia 
Figura $\mathrm{N}^{\circ} 6$ Gráfico respecto de la utilidad tecnología ().

¿Desde su perspectiva, considera que sería útil el uso de recursos tecnológicos, como apoyo didáctico en los procesos de enseñanza aprendizaje?

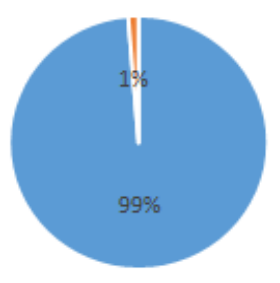

$=\mathrm{Si}=\mathrm{No}$

Fuente: propia

En cuanto al conocimiento de la tecnología de RA un $82 \%$ de los estudiantes encuestados señalan que no la conocen y un 55\% considera que este tipo de recursos didácticos les ayudaría a comprender mejor los conceptos, sobre todo en la asignatura de química considerada como una asignatura de lenguaje difícil por un $59 \%$ de los estudiantes.

Figura $\mathrm{N}^{\circ} 7$ Gráfico respecto del conocimiento de $R A$.

¿Conoce la Tecnología de Realidad Aumentada?

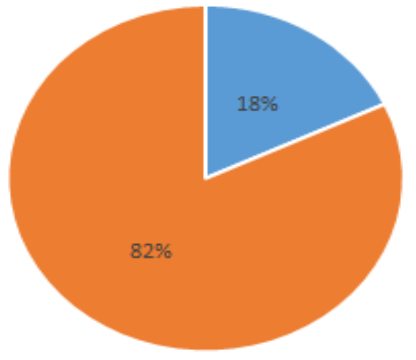

$=\mathrm{Si}=\mathrm{No}$

Fuente: propia

Figura $\mathrm{N}^{\circ} 8$ Gráfico respecto de la comprensión.

De acuerdo a su experiencia, ¿considera que comprende mejor un tema, utilizando modelos tridimensionales?

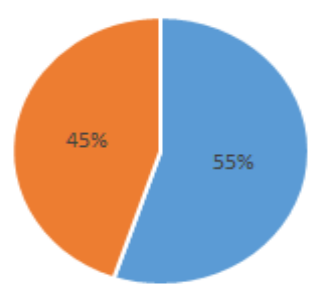

$=\mathrm{Si}=\mathrm{No}$

Fuente: propia 
Figura $\mathrm{N}^{\circ} 9$ Gráfico respecto de la dificultad de la asignatura de química.

¿Ud. considera que la química es un lenguaje

difícil?

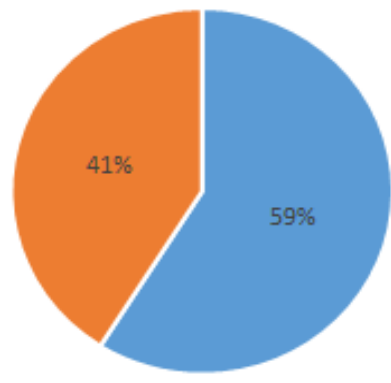

- Si $=$ No

Fuente: propia

$\mathrm{Al}$ observar las respuestas a las preguntas abiertas respecto de la utilidad de utilizar recursos tecnológicos para la enseñanza-aprendizaje de la química, los estudiantes señalaron que al incorporar tecnología les parece una herramienta cercana, un aporte didáctico que facilita el aprendizaje y mejora el método de enseñanza. Algunas de las respuestas se presentan a continuación:

“...Es un apoyo didáctico ya que con la práctica se tiende a aprender mucho más sobre los temas que se ven en aula...”.

“...Es muy útil, debido a que, en el área de las ciencias, sobre todo en química, hay unidades que no se pueden abordar en el laboratorio, debido a que tienen relación a modelos atómicos, geometría molecular u otros, y con estos software es posible trabajar dichos contenidos con mayor facilidad...".

“...Si, porque la enseñanza de las ciencias requiere de la tecnología, hoy en día a medida que avanza la tecnología, existen avances científicos y si hay avances existen mayores recursos para enseñar ciencias como un apoyo didáctico...".

Otro aspecto relevante, que se puede observar, a partir de las respuestas abiertas dice relación con la comprensión de los conceptos y de acuerdo con la experiencia de los propios estudiantes, se les preguntó si consideran que comprenden mejor un tema, utilizando modelos tridimensionales. Las respuestas de los estudiantes indican que aplicar estos recursos didácticos le acercan más a la realidad, el poder visualizar favorece la comprensión del modelo y es fácil asociar a la teoría. Algunas sentencias de los estudiantes:

“...Porque la persona se adaptaría y entendería mejor al ver explicaciones como en la vida real...”.

“...Porque se puede ver en todas las perspectivas posibles...”.

“...Debido a que uno puede observar mejor el tema y aprender más rápido...”. 
“...Porque al ser una forma distinta a los métodos habituales uno tiende a captar más y estar mayormente concentrado...”.

“...Porque los conocimientos son mejores y la información debería ser más verídica...”.

\section{PROPUESTAS DE MEJORA}

Entre las principales propuestas de mejora al estudio está la incorporación de un instrumento de evaluación:

(1) Se sugiere que el instrumento pueda recoger las impresiones de la motivación, los cambios y aprendizajes que se pueden desarrollar producto de la interacción entre pares frente al uso de la tecnología. Se considera que se puede obtener información relevante sobre el impacto de esta interacción en la motivación y por tanto en la mejora de los resultados de aprendizaje en el experimento.

(2) En segundo lugar, se sugiere poder integrar en el instrumento preguntas del tipo cualitativas que permitan obtener otro tipo de información. Esta información cualitativa puede enriquecer el análisis de los resultados del experimento en futuros estudios.

(3) Finalmente, se sugiere buscar formas complementarias de evaluación de carácter formativa, que no necesariamente sean calificativas, lo que podría permitir desarrollar una experiencia diferente $\mathrm{y}$, por tanto, obtener una respuesta distinta de los estudiantes frente al experimento.

\section{CONCLUSIONES}

Se logra diseñar una aplicación móvil con uso de tecnología de RA, utilizando el motor de videojuegos multiplataforma Unity y el SDK de Vuforia, en la temática de Equilibrio ácido-base.

Un porcentaje muy bajo de estudiantes conoce las tecnologías de RA, y de ellos ninguno la relaciona a contextos educativos o de aprendizaje. No obstante, es posible apreciar una muy buena recepción de la estrategia por parte de los estudiantes, lo que podría incidir en una mayor motivación e interés en el proceso de aprendizaje de la química.

La incorporación de recursos didácticos con RA, para la enseñanza de la química, permite a los estudiantes una mejor comprensión del concepto, no es necesario imaginar cómo será, porque a través de la visualización en RA pueden reconocer los elementos tal como son. Al mismo tiempo, al ser una tecnología para ellos familiar no causa tanto rechazo y es de fácil adopción para ser utilizada en entornos de aprendizaje.

Esta propuesta didáctica representa un cambio importante respecto a experiencias pasadas en cursos tradicionales de química, sin embargo, se requiere que el docente disponga de un mayor tiempo de dedicación y constante actualización. 


\section{AGRADECIMIENTOS}

Proyecto de Desarrollo e Innovación Docente en el Pregrado: DID18-0030 “Desarrollo e Implementación de Aplicación Móvil con Tecnología de Realidad Aumentada para la Enseñanza de Química General”, Vicerrectoría de Pregrado, Universidad de La Frontera-Chile. 


\section{REFERENCIAS BIBLIOGRÁFICAS}

Acevedo, D., Cavadia, S., \& Alvis, A. (2015). Estilos de aprendizaje de los estudiantes de la Facultad de Ingeniería de la Universidad de Cartagena (Colombia). Formación Universitaria, 8(4), 15-22. https://scielo.conicyt.cl/scielo.php?pid=S0718-50062015000400003\&script=sci_arttext\&tlng=e

Antezana, V. (2009). La química en la enseñanza de las ingenierías. Journal Boliviana de Ciencias, 6(18).

Horizon, I. (2010). Informe Horizon: Primaria, Secundaria y Enseñanza Universitaria.

Martínez-Hung, H., García-López, A., \& Escalona-Arranz, J. (2017). Modelos de Realidad Aumentada aplicados a la enseñanza de la Química en el nivel universitario. Revista Cubana de Química, 29(1), 1325. http://scielo.sld.cu/scielo.php?script=sci_arttext\&pid=S2224-54212017000100002

Merino, C., Pino, S., Meyer, E., Garrido, J., \& Gallardo, F. (2015). Realidad aumentada para el diseño de secuencias de enseñanza-aprendizaje en química. Educación Química, 26(2), 94-99. https://www.sciencedirect.com/science/article/pii/S0187893X15000051

Saidin, N., Halim, N., \& Yahaya, N. (2015). A review of research on augmented reality in education: Advantages and applications. International Education Studies, 8(13), 1-8. http://citeseerx.ist.psu.edu/viewdoc/download?doi=10.1.1.730.8456\&rep=rep1\&type=pdf

Torres, M. (2010). La enseñanza tradicional de las ciencias versus las nuevas tendencias educativas. Revista Electrónica Educare, 14(1), 131-142. https://www.redalyc.org/pdf/1941/194114419012.pdf 